\title{
Zur Emeritierung von Prof. Dr. med. Michael Molls - Direktor der Klinik und Poliklinik für Strahlentherapie und Radiologische Onkologie, TU München
}

\author{
Anca-L. Grosu
}

Online publiziert: 10. Oktober 2014

(C) Springer-Verlag Berlin Heidelberg 2014

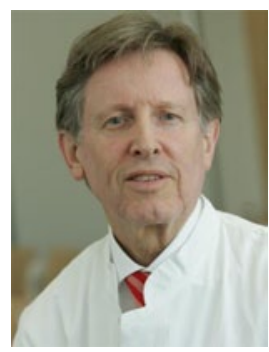

Michael Molls (Mit freundlicher Genehmigung)

Michael Molls ist im Schwarzwald geboren und aufgewachsen. Der Vater war Landarzt in Hornberg. Michael hat zwei Brüder und eine Schwester. Das Gymnasium hat er im Hausach besucht, studiert hat er an der Medizinische Fakultät in Freiburg.

Nach dem Studium geht Michael Molls in die weite Welt - NY? „Nö, er kann mich mal nach Istanbul schicken“ (Wilhelm Genazino) Er geht nach Istanbul: „sterbende Schöne“, „teuflischer Lärm, göttliche Stille“, „Märchen vor der Haustür“ (Wilhelm Genazino). Später ist er im ehemaligen Jugoslawien. Er war zwei Jahre in beiden Ländern als Arbeitsmediziner tätig. Michael Molls heiratet Jasmina, sie haben 2 Kinder.

Zurück in Deutschland arbeitet er zwischen 1974-76 als Assistent am Pharmakologischen Institut der Universität Freiburg, wo er zum Thema ,Anaphylaktischer Schock und

Laudatio zur Ernennung von Michael Molls zum Ehrenmitglied der Deutschen Gesellschaft für Radioonkologie, Düsseldorf, Juli 2014

Prof. Dr. A.-L. Grosu ( $\square)$

Klinik für Strahlenheilkunde, Universitätsklinikum Freiburg, Freiburg, Deutschland

E-Mail: anca.grosu@uniklinik-freiburg.de

dessen therapeutische Beeinflussbarkeit“" promoviert. 1976 verlässt Michael Molls Freiburg, Schwarzwald, Istanbul, Balkan, um reif und erwachsen die Kraft und die Ruhe zu finden, „eine Sache rund zu formen und in Gelassenheit etwas Ganzes daraus zu schmieden “ (Thomas Mann: Tonio Kröger).

Er wird zunächst Wissenschaftlicher Mitarbeiter am Institut für Medizinische Strahlenbiologie des Universitätsklinikums Essen unter der Leitung von Prof. Streffer. Hier arbeitet er an der Etablierung des In-vitro-Modells des Präimplantationsembryos der Maus und macht an diesem Modell sowohl embryologische als auch strahlenbiologische Untersuchungen, die einem besseren Verständnis der Strahlen-Teratogenese, vor allem aber der Frage des radiogenen Zelltodes dienen. Aus diesen stammen die ersten Publikationen in internationalen Journalen (International Archive of Allergy and Applied Immunology, Cell and Tissue Kinetics, Radiation Research und International Journal of Radiation Biology and Related Studies in Physics, Chemistry and Medicine).

Es folgt die klinische Arbeit an der Strahlenklinik des Universitätsklinikums Essen unter Führung von Prof. Scherer und später von Prof. Sack. Fünf in Deutschland ansässige Klinikdirektoren stammen aus der radioonkologischen Essener Schule, einer davon ist Michael Molls. Habilitiert in Embryologie, Strahlenbiologie und in klinischer Radioonkologie und mit einer breiten klinischen und strahlenbiologischen Erfahrung übernimmt er als Ordinarius und Direktor die Klinik und Poliklinik für Strahlentherapie und Radiologische Onkologie des Klinikums Rechts der Isar der Technischen Universität als Nachfolger von Alfred Breit. Beide sind später eng befreundet. Unter Molls leuchtet die Klinik: Forschungsprojekte und hochwertige Patientenbehandlung! Drei Säulen: Klinik, Biologie und Physikalisch-Technologische-Entwicklung bestimmen die 
Forschungsaktivitäten am Klinikum Rechts der Isar über mehr als zwei Jahrzehnte.

Im klinischen Sektor ist die Gruppe national und international geachtet in Bereichen wie Präzisionsstrahlentherapie (Radiochirurgie und Stereotaktische Strahlentherapie im Kopf und Körperbereich, Intensitätsmodulierte Strahlentherapie, IMRT, Tomotherapie). Die Klinik initiiert multizentrische DFG- und BMBF-geförderte klinischen Studien. Im experimentellen Bereich dominieren Themen wie Hypoxie, Strahlentherapie und Immunologie oder Strahlentoleranz von Normalgewebe.

Die Technologische Entwicklung und die konstruktive Kooperation mit anderen technologisch-physikalisch orientierten Disziplinen war immer ein wichtiges Ziel des Duos Molls/Kneschaurek (Prof. Dr. Peter Kneschaurek, Leiter der Medizinischen-Physik an der Klinik für Strahlentherapie, Klinikum rechts der Isar, 1992-2013). Das DFG-Exzellenzcluster „Munich-Centre for Advanced Photonics“, in dem Molls als Ko-Sprecher für die Bio-Medizin verantwortlich ist, mit ca. 60 Mio. $€$ in zwei Förderperioden dotiert, krönt seine Aktivitäten. In diesem Exzellenzcluster wird auf der Basis von Laser und Photonenphysik nach neuen hochpräzisen strahlentherapeutischen und diagnostischen Methoden geforscht. Aus dem Center heraus ist es vor einigen Jahren gelungen, einen Forschungsbau mit Instrumenten für die beiden Münchener Universitäten einzuwerben: Center of Advanced Laser Application (CALA).

Michael Molls ist Autor von über 300 Originalarbeiten, Mitherausgeber der deutsch-amerikanischen Buchreihe „Medical Radiology - Radiation Oncology“ im Springer Verlag, Mitherausgeber des zweibändigen Lehrbuches „Radioonkologie“ im Zuckschwerdt Verlag und auch Mitherausgeber von Blood Perfusion and Microenvironment of Human Tumors, Implications for Clinical Radiooncology (Springer); 3-D Radiation Treatment: Technological Innovations and Clinical Results (Karger); The Impact of Tumor Biology on Cancer Treatment and Multidisciplinary Strategies (Springer).

Michael Molls hat über die Jahre verschiedene Funktionen ausgeübt und diverse Auszeichnungen erhalten:

- Stellvertretender Sprecher der Emeriti of Excellence der TUM (seit 2014)

- Mitglied des wissenschaftlichen Beirates für die „ELI Beamlines Facility“ Prag (Extreme Light Infrastructure, European Project) (seit 2010)

- Mitglied des Vorstandes (seit 2006) und Ko-Sprecher (seit 2010) des DFG-Exzellenzclusters „Munich Centre for Advanced Photonics (MAP)“, verantwortlich für die Biomedizin

- Projektleiter des Strahlenbiologie-Projektes „CARDIORISK“ (EU, 7. Rahmenprogramm) (2008-2011)

- Mitglied des Peer Review College of the Danish Council for Strategic Research (2008)
- Mitglied des Fachkollegiums Medizin der DFG (Krebsforschung und Medizin-Technik) (2007-2012)

- Präsident der Akademie für Fort- u. Weiterbildung der „Deutschen Gesellschaft für Radioonkologie“ (2004-2009)

- Mitglied des Vorstandes (1999-2003) und Mitglied des Aufsichtsrates (2003-2009) des Klinikums rechts der Isar

- Vorsitzender des TZM (Tumorzentrum München) an der LMU und der TUM (2001-2003)

- Mitglied des Wissenschaftlichen Direktoriums des Forschungsreaktors der TUM (1996-2003)

- Präsident der „Deutschen Gesellschaft für Radioonkologie“(1997-1999)

- Mitglied von Expertenkommissionen (Wissenschaftsrat, DFG, Helmholtz Gemeinschaft, Bundes- und Landesministerien) und Beiräten

- Mitglied nationaler und internationaler wissenschaftlicher Gesellschaften

- Hanns-Langendorff-Preis (1979)

- Mitglied der Deutschen Akademie der Naturforscher Leopoldina (seit 1998)

- Präsident des gemeinsamen Jahreskongresses der Deutschen und Österreichischen Gesellschaften für Radioonkologie und der Deutschen Gesellschaft für Medizinische Physik (2000)

- Ehrenmitglied der Österreichischen und Deutschen Gesellschaften für Radioonkologie (2004, 2014)

- Ehrenurkunde des Tumorzentrums München (2007)

Die Klinik ist unter seiner Leitung gewachsen! Er hat uns alle auf Trapp gebracht und die „Ohren ganz schön lang gezogen“ (Zitat Molls), wenn wir unsere Arbeit „nicht ordentlich“ gemacht hatten. Michael Molls hat getobt und gelobt, gedroht und gewürdigt, und zum Schluss haben wir ihn alle geliebt und wir waren ihm unendlich dankbar. Als Chefin in Freiburg denke ich sehr oft an die Münchener Zeiten und frage mich immer wieder: wie hat er das nur geschafft? Wir, seine Schüler, glauben heute, dass er uns durch seine Strenge und durch seine Gelassenheit, durch seine Ernsthaftigkeit und Heiterkeit beigebracht hat, Tag für Tag in Freude und Ruhe ,eine Sache rund zu formen und in Gelassenheit etwas Ganzes daraus zu schmieden ". Diese Lehre tragen wir in unseren Herzen und in unseren Köpfen. Und etwas davon geben wir Tag für Tag unseren Lehrlingen weiter: Sechs Chefärzte in Fulda (Feldmann), in Basel (Zimmermann), in Linz (Geinitz,), in Bodo/Norwegen (Nieder), in Krems (Beyerl) und ich in Freiburg.

Bach? Nein - lass uns Mozart spielen. Denn: „Ich habe die Vermutung..., ich sei nicht schlechthin sicher, ob die Engel, wenn sie im Lobe Gottes begriffen sind, gerade Bach spielen - ich bin aber sicher, dass sie, wenn sie unter sich sind, Mozart spielen und dass ihnen dann doch auch der liebe Gott besonders gerne zuhört.“ (Karl Barth) 